\title{
Detección de anticuerpos anti-Toxocara spp. en caninos de una comunidad periurbana de la provincia de Heredia, Costa Rica
}

\section{Detection of anti-Toxocara spp. antibodies in canines from a peri-urban area in the province of Heredia, Costa Rica}

\section{Detecção de anticorpos anti-Toxocara spp. em caninos de uma comunidade periurbana na província de Heredia, Costa Rica}

Natalia Guzmán-Gómez ${ }^{1 凶}$, F. Raúl Bermúdez-Salas ${ }^{1}$, Luis José Schmidt ${ }^{1}$, Marta C. Bonilla², Gaby Dolz $^{2}$, Andrea Urbina-Villalobos ${ }^{1}$

1. Laboratorio de Zoonosis, Escuela de Medicina Veterinaria, Universidad Nacional, Heredia, Costa Rica. E-mail: natimichelle11@gmail.com; rbs.vetbass@gmail.com ; luisjoschmidt@gmail.com ; andreaurbina3@gmail.com

2. Laboratorio de Docencia e Investigación en Medicina Poblacional, Programa MEDPOB, Escuela de Medicina Veterinaria, Universidad Nacional, Heredia, Costa Rica.Email: mabongo27@gmail.com, gaby.dolz.wiedner@una.ac.cr

Recibido: 21 de agosto de 2019 Corregido: 6 de febrero de 2020 Aceptado: 25 de febrero de 2020

\section{Resumen}

La toxocariasis es una enfermedad zoonótica cosmopolita causada por nemátodos del género Toxocara. Los perros son hospedadores definitivos de Toxocara canis y pueden ser asintomáticos o presentar signos clínicos inespecíficos. El ser humano puede infectarse y desarrollar diferentes patologías.

En Costa Rica, se ha realizado estudios de prevalencia de huevecillos de Toxocara spp. en muestras de suelo y heces de caninos, pero no se ha investigado la prevalencia de anticuerpos contra este parásito en perros. Este estudio tiene como objetivo analizar 55 sueros de caninos de una comunidad periurbana de Heredia, Costa Rica, utilizando una prueba comercial para la detección de anticuerpos IgG anti-Toxocara spp. Además, se utilizó la información obtenida, mediante un cuestionario aplicado a las personas poseedoras de los perros, para conocer las prácticas de manejo con sus mascotas y el conocimiento sobre enfermedades zoonóticas. Se determinó una seroprevalencia de $29 \%$, lo cual evidencia que los caninos se infectaron con este parásito en alguna etapa de su vida. Se comprueba un desconocimiento generalizado acerca de las enfermedades zoonóticas por parte de los propietarios de los caninos; la mayoría de ellos aplicaba prácticas de manejo inadecuadas, tales como: no recoger las heces de sus perros en sitios públicos, poca o ninguna atención veterinaria para sus mascotas y protocolos de desparasitación de sus animales sin guía profesional ni registros confiables, lo cual podría favorecer la infección de sus mascotas con parásitos zoonóticos.

Palabras clave: Toxocara spp., toxocariasis, perros, zoonosis, seroprevalencia

\section{Abstract}

Toxocariasis is a cosmopolitan zoonotic disease caused by nematodes belonging to the genus Toxocara. Dogs are the definitive hosts of Toxocara canis and can develop non-specific clinical symptoms or remain asymptomatic. Humans can also become infected and develop different pathologies.

$\square$

Correo de correspondencia: natimichelle11@gmail.com 
Prevalence studies on Toxocara spp. eggs in soil samples and canine feces were conducted in Costa Rica; however, the prevalence of antibodies against this parasite in dogs has not been investigated yet. This study aimed to analyze 55 canine sera from a peri-urban community in Heredia, Costa Rica, using a commercial test for the detection of IgG antibodies against Toxocara spp. In addition, dog owners were asked to fill out a questionnaire to know pet management practices and verify their knowledge of zoonotic diseases.

A $29 \%$ seroprevalence was determined, evidencing that canines had been infected with this parasite at some point in their lives. A general lack of knowledge about zoonotic diseases was determined among the owners, and most of them showed inadequate pet management practices such as not collecting canine feces in public places, little or no veterinary care, and deworming protocols without professional guidance or reliable records which could favor infection with zoonotic parasites.

Keywords: Toxocara spp., toxocariasis, dogs, zoonoses, seroprevalence

\begin{abstract}
Resumo
A toxocaríase é uma doença zoonótica cosmopolita causada por nematóides do gênero Toxocara. Os cães são hospedeiros definitivos do Toxocara canis e podem ser assintomáticos ou mostrar sinais clínicos inespecíficos. O ser humano pode ser infectado e desenvolver diferentes patologias.

Em Costa Rica, foram realizados estudos de prevalência de ovos de Toxocara spp. em amostras de solo e fezes de cães, mas a prevalência de anticorpos contra esse parasita em cães ainda não tem sido investigado. Este estudo tem como objetivo analisar 55 soros caninos de uma comunidade periurbana de Heredia, Costa Rica, utilizando um teste comercial para a detecção de anticorpos IgG anti-Toxocara spp. Além disso, as informações obtidas, através de um questionário aplicado aos donos dos cães, foram utilizadas para conhecer as práticas de manejo com seus animais de estimação e o conhecimento sobre doenças zoonóticas.

Foi determinada uma soroprevalência de $29 \%$, o que mostra que os caninos foram infectados com esse parasita em algum estágio de sua vida. Existe um desconhecimento geral sobre doenças zoonóticas por parte dos proprietários caninos; a maioria deles aplicava práticas inadequadas de manejo, tais como: não coletar as fezes de seus cães em locais públicos, pouca ou nenhuma atenção veterinária para seus animais de estimação e protocolos de desparasitação para seus animais sem orientação profissional ou registros confiáveis, o que poderia favorecer a infecção de seus animais de estimação com parasitas zoonóticos.
\end{abstract}

Palavras-chave: Toxocara spp., Toxocaríase, cães, zoonose, soroprevalência

\title{
Introducción
}

La toxocariasis es la infección causada por nemátodos del género Toxocara. Existen diversas especies, pero no todas se consideran zoonóticas (Center for Food Security \& Public Health 2016). Toxocara canis tiene, como hospedero definitivo, a los cánidos y es uno de los helmintos intestinales de caninos más importante pues se reportan casos de infección a nivel mundial. Se considera uno de los principales causantes de la toxocariasis humana (Schnieder et al. 2011; Center for Food Security \& Public Health 2016).

Los caninos adquieren la infección con T. canis por: vía transplacentaria, transmamaria, ingesta de huevos infectantes, directamente del ambiente y consumo de larvas enquistadas en tejidos de hospederos paraténicos (Schnieder et al. 2011). Los perros pueden desarrollar una infección latente con larvas enquistadas en diversos tejidos o una infección patente con excreción de huevos no embrionados en las heces, los cuales bajo condiciones favorables se vuelven infectantes en 9-15 días y pueden permanecer viables en el suelo por varios años, constituyendo el origen de infección para otros cánidos y seres humanos (Schnieder et al. 2011; Rojas-Salamanca et al. 2016). Los animales pueden ser asintomáticos o 
presentar signos clínicos inespecíficos como: diarrea, vómito, distención abdominal, pelo hirsuto, entre otros (Calderón et al. 2008; Willard 2014).

La toxocariasis humana es considerada una de las cinco enfermedades parasitarias más desatendidas a nivel mundial, pues a pesar de su impacto en la salud pública, no existen protocolos de vigilancia y prevención (Centers for Disease Control and Prevention 2017). Las personas infectadas desarrollan el síndrome de larva migrans que puede ser: visceral (LMV), ocular (LMO), neurológico o encubierto. La población infantil es la más propensa a infectarse (Rojas-Salamanca et al. 2016).

En Costa Rica, se desconoce el estado actual de la toxocariasis en los habitantes. En el servicio de oftalmología del Hospital Nacional de Niños (HNN) se atiende, anualmente, 6 a 7 niños con ceguera parcial por LMO (Rojas 2015). Entre 1986 y 1993 se analizaron en Costa Rica, mediante la prueba de ELISA empleando antígeno excretor-secretor de larvas de T. canis, 282 sueros de niños hospitalizados con diagnóstico clínico de eosinofilia, dolor abdominal en estudio o Larva Migrans Visceral. Se determinó una tasa de anticuerpos anti-Toxocara canis de 48.6\% (Dra. Andrea Urbina V., Laboratorio de Zoonosis de la Universidad Nacional, comunicación personal).

En caninos, esta parasitosis se diagnostica combinando hallazgos clínicos con pruebas de laboratorio. El examen coproparasitológico es la prueba más utilizada por los veterinarios. Sin embargo, un resultado negativo no excluye la infección, pues no todos los perros desarrollan una infección patente. Los cachorros muy parasitados pueden presentar síntomas antes de la aparición de huevos en las heces (Fernández et al. 2008; Willard 2014).

La prevalencia mundial de huevecillos de T. canis en heces de caninos es de 2.9\%-44.3\% (Overgauuw 1997; Silva-Regis et al. 2011). En Costa Rica se reporta en un rango de 6.0\%-25.3\% (Arguedas-Zeledón et al. 2006; Paquet-Durand et al. 2007; Calderón et al. 2008; Castro et al. 2009; Sáenz-Ugalde 2013). Estas cifras podrían estar subestimadas pues no todos los animales infectados eliminan huevos del parásito en las heces; aún en infecciones patentes la excreción de huevecillos puede ser intermitente (Silva-Regis et al. 2011; Center for Food Security \& Public Health 2016).

Existen pruebas serológicas para la detección de anticuerpos contra T. canis, las cuales permiten identificar los animales que han tenido contacto con el parásito, aunque no desarrollen una infección patente (Silva-Regis et al. 2011). Los estudios de seroprevalencia en perros son escasos; A nivel mundial se reportan seroprevalencias entre 66.7\% y 84.9\% (Martínez-Barbabosa et al. 2008; Silva-Regis et al. 2011; García et al. 2014). En Costa Rica, no se ha realizado investigaciones de este tipo, por lo cual, el objetivo de este estudio consiste en determinar la presencia de anticuerpos contra Toxocara spp. en caninos de una comunidad periurbana de la provincia de Heredia, Costa Rica. Además, contribuir con el conocimiento de la situación de esta parasitosis en la población canina.

\section{Materiales y métodos}

\section{Tipo de estudio}

Se realiza un estudio transversal descriptivo, en muestras de suero de caninos de una comunidad periurbana de Costa Rica. 


\section{Procedencia de las muestras y datos de los caninos}

Para esta investigación, se utilizó un banco de 55 sueros caninos, el cual se creó en 2016 para un estudio serológico sobre tripanosomiasis canina, en el cual se incluye perros de cualquier edad y sexo, con propietarios anuentes a contestar un cuestionario y otorgar el consentimiento para recolectar la muestra de sangre. La información recolectada en esos cuestionarios se registró en una base de datos de Excel, a la cual se tuvo acceso. Se contó con datos sobre: edad, sexo, raza y esterilización de cada canino, así como el número de perros en cada casa. Este registro incluyó también información suministrada por los tutores de 46 caninos acerca del conocimiento sobre enfermedades zoonóticas y prácticas de manejo que aplicaban a sus perros, tales como: alimentación brindada, esquemas de control veterinario y disposición de las heces (en casa y sitios públicos). Nueve propietarios de perros no fue posible localizarlos para completar el cuestionario (Bonilla et al. 2018).

Los sueros se mantuvieron almacenados a $-20^{\circ} \mathrm{C}$ en la Escuela de Medicina Veterinaria (EMV) de la Universidad Nacional de Costa Rica (UNA) hasta ser utilizados nuevamente en este estudio.

Los 55 caninos eran del distrito de Getsemaní, una comunidad periurbana, perteneciente a los cantones de Barva y San Rafael, en la provincia de Heredia (Municipalidad de Barva 2014; Instituto de Desarrollo Rural de Costa Rica 2016).

\section{Cálculo del margen de error}

Se calculó el margen de error (d) utilizando la fórmula de la Figura 1, con un valor de Distribución $\mathrm{Z}$ para 95\% de confianza (Z) de 1.96; un tamaño de muestra (n) de 55 caninos y una población estimada (N) de 1663479 caninos en el área central del país (World Animal Protection 2016; Instituto Nacional de Estadística y Censos 2018). La prevalencia más baja reportada de Toxocara spp. en heces de caninos es de 6\% (Sáenz-Ugalde 2013), por lo cual, se estimó una prevalencia mínima de anticuerpos (p) de $10 \%$ pues no todos los perros desarrollan infecciones patentes al entrar en contacto con el parásito y los anticuerpos persisten en el tiempo (Center for Food Security \& Public Health 2016). Por último, se obtuvo un valor de 0.90 para la variable "q" (1-p), resultando un margen de error de $7.9 \%$.

$$
\mathrm{d}=\sqrt{\frac{N \times Z_{\alpha}^{2} \times p \times q}{n \times(N-1)+Z^{2}{ }_{\alpha} X p \times q}}
$$

Figura 1. Fórmula para calcular el margen de error.

\section{Análisis serológico}

Las muestras se analizaron utilizando el kit comercial VetLine Toxocara (Novatec Inmunodiagnostica $\mathrm{GmbH}^{\circledR}$ ), un ensayo enzimático inmunoadsorbente (ELISA) cualitativo que detecta la presencia de anticuerpos IgG anti-Toxocara spp. en suero de mamíferos. No se especifica el tipo de antígeno que utiliza la prueba, pero tiene una sensibilidad de $90.3 \%$ y una especificidad de $91.2 \%$ para sueros caninos. (NovaTec Inmunodiagnostica GmbH 2016). 
Se siguieron las instrucciones del fabricante para el desarrollo de la prueba y la interpretación de los resultados. Las absorbancias se establecieron mediante el lector de microplacas Multiskan EX de Thermo Fischer Scientific Inc., empleando un filtro de $450 \mathrm{~nm}$.

\section{Clasificación del nivel de cuidado de los caninos por parte de los propietarios}

La tenencia responsable de mascotas involucra muchos factores; sin embargo, con base en la información disponible, de 46 caninos y sus dueños, se eligió 5 variables reconocidas por la American Veterinary Medical Association (AVMA) (2019) como criterios importantes para evaluar la responsabilidad de un propietario con sus animales. Estos criterios son: uso de productos desparasitantes, frecuencia de desparasitación, frecuencia de visitas al veterinario, recolección de heces en el hogar y recolección de heces en áreas públicas. Estas variables se agruparon en un solo indicador, denominado nivel de cuidado de mascotas.

Las prácticas reportadas por los dueños se distribuyeron en cada variable, y se catalogaron como: satisfactorias, aceptables y no aceptables. Para realizar esta clasificación se tomó como base las guías y recomendaciones brindadas por la Dra. Cheryl Yuill, el European Scientific Counsel Companion Animal Parasites (ESCCAP) y el Tropical Council for Companion Animal Parasites (TroCCAP) (Yuill 2011; European Scientific Counsel Companion Animal Parasites 2017; Tropical Council for Companion Animal Parasites 2017). Se clasificó el nivel de cuidado de las mascotas como: nulo, bajo, medio y alto.

\section{Resultados}

Los 55 caninos analizados tenían propietario. Se encontraban en un rango de edad de 1-14 años, con una media de 4 años. Estaban distribuidos en 29 viviendas, por lo que había un promedio de 1.89 perros/casa. Un total de 29 (53\%) eran machos y 26 (47\%) eran hembras, 36 (65\%) eran caninos sin raza definida (SRD) y 19 (35\%) eran de raza pura. Había ocho hembras y 19 machos sin castrar.

Del total de sueros analizados, 16 (29\%) resultaron positivos a anticuerpos IgG anti-Toxocara spp., 37 (67\%) fueron negativos y dos (4\%) dieron resultados no concluyentes (Gráfico 1).

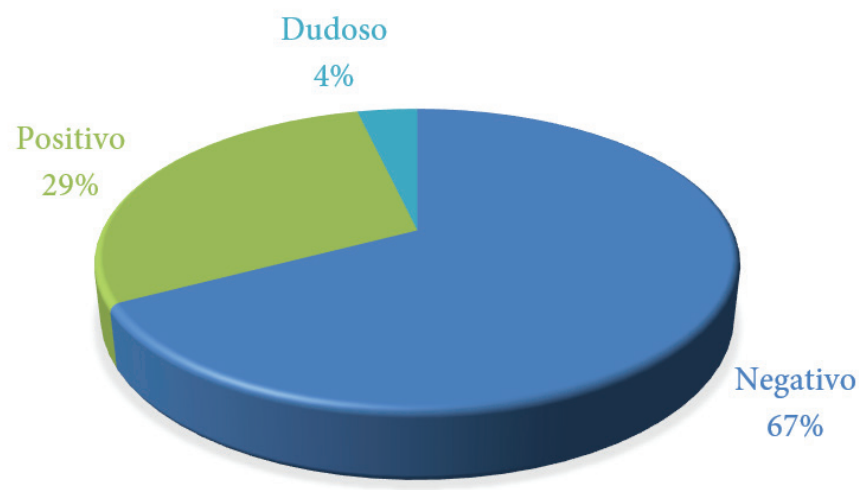

Gráfico 1. Resultado de la prueba de ELISA para detección de anticuerpos IgG anti-Toxocara spp. en sueros de 55 caninos. 
Entre los 16 caninos seropositivos había ocho hembras y ocho machos. El 75\% (12/16) tenía 1-6 años. El 33\% (12/36) de los caninos SRD y el 21\% (4/19) de los perros con raza pura tenían anticuerpos contra Toxocara spp. (Cuadro 1). De las hembras seropositivas, tres se encontraban sin castrar, con edades entre 2 y 5 años.

Cuadro 1. Distribución de los caninos positivos a anticuerpos IgG anti-Toxocara spp. según sexo, edad y raza.

\begin{tabular}{|c|c|}
\hline \multicolumn{1}{|c|}{ Variable } & Positivos/ total de caninos (\%) \\
\hline Sexo & $8 / 29(28)$ \\
\hline Macho & $8 / 26(31)$ \\
\hline Hembra & \\
\hline Edad $\quad$ & $8 / 29(28)$ \\
\hline 4-6 años & $4 / 18(22)$ \\
\hline $7-9$ años & $2 / 4(50)$ \\
\hline $10-12$ años & $2 / 3(66)$ \\
\hline $13-15$ años & $0 / 1(0)$ \\
\hline Raza & $12 / 36(33)$ \\
\hline SRD & $4 / 19(21)$ \\
\hline Pura & \\
\hline
\end{tabular}

En el 31\% de las viviendas (9/29) se encontró caninos seropositivos. En estas 9 casas había más de un perro como mascota. En el 44\% (4/9) de las viviendas se encontró más de un canino positivo y en un $88 \%$ (8/9) convivían animales positivos y negativos. En todas las casas con tres o más perros, un promedio del 53\% de los caninos presentó anticuerpos circulantes contra Toxocara spp. (Gráfico 2).

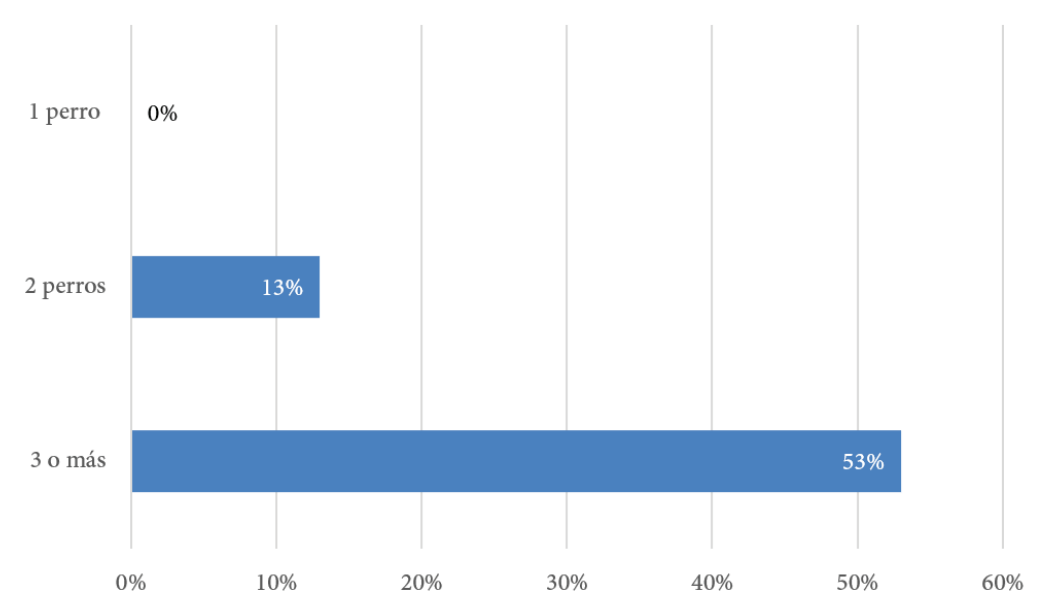

Gráfico 2. Porcentaje promedio de caninos seropositivos a Toxocara spp. en cada casa según la cantidad de perros mascota. 
El cuestionario fue contestado por 25 dueños de 46 caninos. De esta manera, se obtuvo información sobre el manejo de 12 perros positivos, 33 negativos y uno dudoso. Ninguno de los 25 propietarios encuestados conocía sobre enfermedades transmitidas entre animales y personas.

En el cuadro 2, se muestra las frecuencias de caninos seropositivos, según las diferentes prácticas de manejo reportadas por los 25 encargados. Un total de 27 caninos eran alimentados solamente con concentrado; los 19 restantes con una mezcla de alimento casero y comercial. En ambos esquemas de dieta, un $26 \%$ de los animales presentó anticuerpos anti-Toxocara spp. El 65\% (26/46) de los perros no era sometido a revisiones veterinarias periódicas. Sin embargo, la mayoría de los animales positivos, (9/12) se reportó que tenía chequeos veterinarios anuales. Respecto a la desparasitación, el 89\% (42/46) de los caninos se desparasitaba periódicamente, incluyendo los 12 perros seropositivos. Solamente uno de los propietarios pudo aportar el nombre del antiparasitario utilizado. El 50\% (23/46) de los animales se desparasitaba trimestralmente. La mitad (6/12) de los perros positivos se encontraba bajo este esquema.

Cuadro 2. Distribución de los perros seropositivos a Toxocara spp. según algunas prácticas de manejo reportadas por los dueños de 46 caninos.

\begin{tabular}{|l|c|}
\hline \multicolumn{1}{|c|}{ Variable } & Positivos/ total de caninos (\%) \\
\hline Tipo de alimentación & \\
\hline Concentrado & $7 / 27(26 \%)$ \\
\hline Casera y concentrado & $5 / 19(26 \%)$ \\
\hline Visitas al veterinario & $3 / 13(23 \%)$ \\
\hline Nunca & $9 / 20(45 \%)$ \\
\hline 1 vez al año & $0 / 13(0 \%)$ \\
\hline Sólo cuando enferma & \\
\hline Desparasitación & $12 / 42(29 \%)$ \\
\hline Sí & $0 / 4(0 \%)$ \\
\hline No & \\
\hline Desparasitante utilizado (n= 42) & $0 / 1(0 \%)$ \\
\hline Conoce el nombre & $12 / 41(29 \%)$ \\
\hline Desconoce/No respondió & \\
\hline Frecuencia de desparasitación $(\mathbf{n}=\mathbf{4 2})$ & $0 / 2(0 \%)$ \\
\hline Mensual & $6 / 23(26 \%)$ \\
\hline Trimestral & $2 / 6(33 \%)$ \\
\hline Semestral & $4 / 10(40 \%)$ \\
\hline Anual & $0 / 1(0 \%)$ \\
\hline No respondió &
\end{tabular}

Los dueños del 74\% (34/46) de los perros afirmaron recoger las heces de sus mascotas en el hogar, pero los propietarios del 33\% (15/46) de los caninos reportaron recolectarlas también en áreas públicas. El $42 \%$ (7/12) y 35\% (11/31) de los animales que no les recogen las heces, en la casa o en áreas públicas respectivamente, resultaron seropositivos (Gráfico 3). 
Rev. Ciencias Veterinarias, Vol. 38, $\mathrm{N}^{\circ}$ 1, [28-42], E-ISSN: 2215-4507, enero-junio, 2020

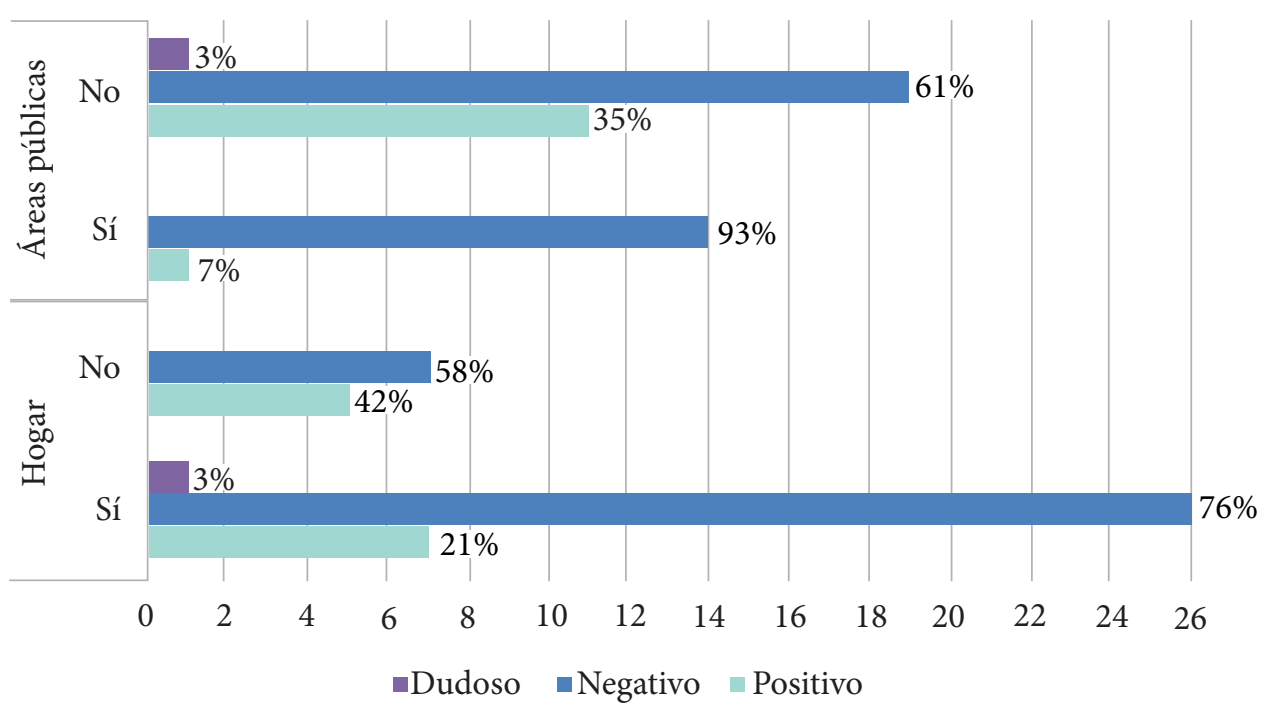

Gráfico 3. Distribución de los resultados serológicos a Toxocara spp. de 46 caninos, según la recolección de heces en el hogar y áreas públicas.

Según la clasificación del nivel de tenencia responsable, por parte de los dueños, el 15\% (7/46) de los caninos tenía un nivel de cuidado bajo; un 46\% (21/46) un nivel medio y un 37\% (17/46) un nivel alto; con un 33\% (4/12) de los animales seropositivos en cada nivel. Solamente uno de los animales (2\%) tenía un nivel de atención nulo por parte de sus propietarios, el cual resultó seronegativo. El perro con resultado dudoso recibía un nivel de cuidado medio (Gráfico 4).

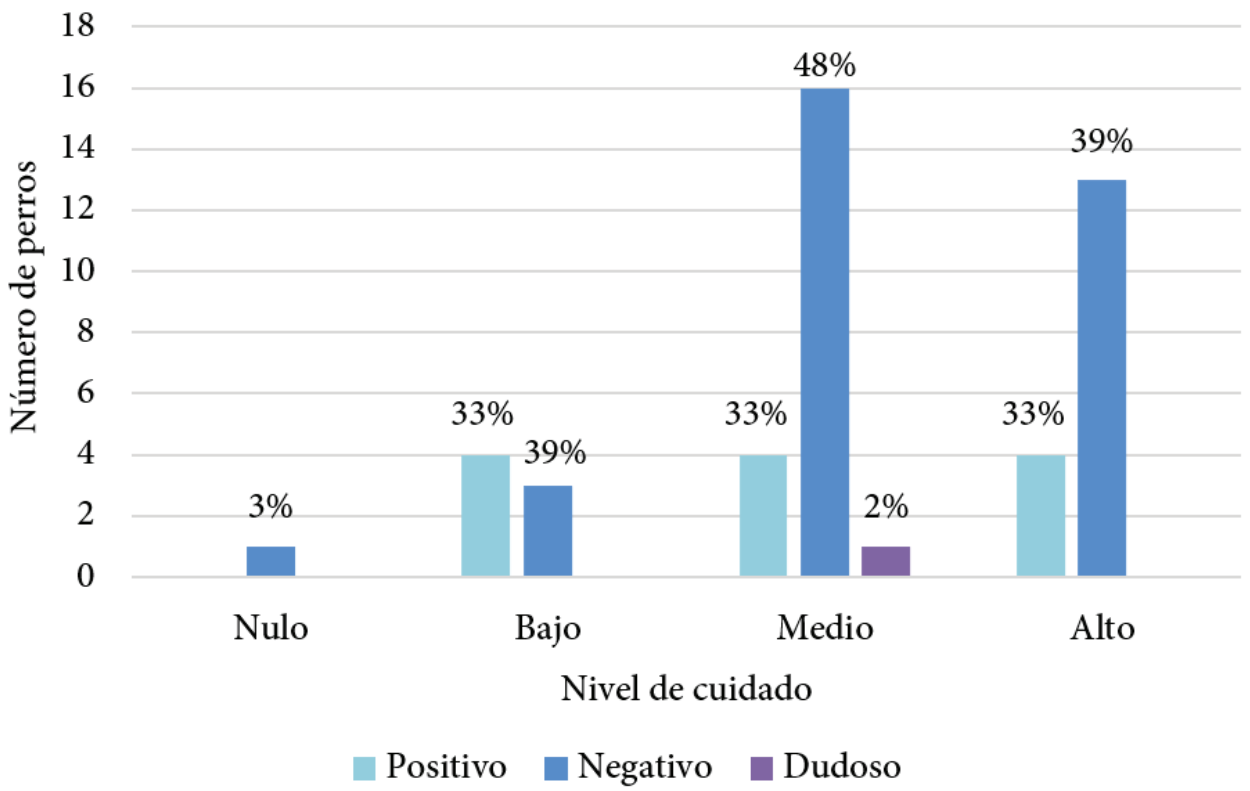

Gráfico 4. Distribución de los caninos con resultados serológicos positivos, negativos y dudosos a Toxocara spp. según el nivel de cuidado por parte de los propietarios. 


\section{Discusión}

El diagnóstico preciso de la toxocariasis canina es esencial para la salud veterinaria y pública. El examen coproparasitológico es el método más utilizado por los veterinarios para el diagnóstico de parásitos gastrointestinales, y la técnica de flotación la más utilizada (Fernández et al. 2008). La mayoría de las investigaciones sobre toxocariaisis, en caninos, se han basado en el examen coproparasitológico; sin embargo, un resultado negativo no descarta que el animal pueda tener una infección activa (Silva-Regis et al. 2011).

Se ha desarrollado diversas pruebas inmunodiagnósticas, El ELISA es la prueba más utilizada para la detección de anticuerpos anti-Toxocara spp. Si bien la detección de inmunoglobulinas circulantes no diferencia entre una infección reciente o pasada, sí evidencia que hubo contacto con el parásito, lo cual es información valiosa para la práctica clínica y estudios epidemiológicos (Watthanakulpanich 2010).

En esta investigación sólo se dispuso de sueros almacenados desde 2016. En esa oportunidad no se recolectó muestras de heces. A pesar de lo anterior, la prevalencia de anticuerpos anti-Toxocara spp. resultó cinco veces mayor que la prevalencia de huevecillos más baja reportada en muestras fecales en nuestro país (Sáenz-Ugalde 2013), lo cual concuerda con lo documentado en otras latitudes (Silva-Regis et al. 2011).

La diferencia en los porcentajes de seroprevalencia en caninos de diferentes países puede deberse a distintos factores socioeconómicos y geográficos, propios de cada región analizada, los cuales inciden sobre las prevalencias del parásito en los animales y personas, así como a posibles variaciones de sensibilidad y especificidad, según la prueba de inmunodiagnóstico empleada (Watthanakulpanich 2010; Rojas-Salamanca et al.2016). Costa Rica es un país en desarrollo, con condiciones socioeconómicas desfavorables en diferentes zonas del territorio, posee una amplia población de perros callejeros y las estrategias para educar a la población sobre buenas prácticas sanitarias y tenencia responsable de mascotas son deficientes. Estos aspectos son factores de riesgo para la infección con Toxocara spp. en caninos y humanos (Silva-Regis et al. 2011; Rojas-Salamanca et al. 2016), por lo cual, no se puede descartar que la seroprevalencia en perros de otras partes del país sea más elevada.

En este estudio, se encontró dos animales con resultados no concluyentes. Si bien el ELISA utilizado tiene una sensibilidad y especificidad confiable, existen variables que pueden afectar el resultado, tales como: mal almacenamiento y manipulación de las muestras y reactivos, reacciones cruzadas de anticuerpos y, presencia de interferencias en el suero como ictericia, hemólisis y lipemia (NovaTec Inmunodiagnostica $\mathrm{GmbH}$ 2016). Además, los animales en etapas tempranas de la infección pueden dar resultados dudosos o falsos negativos; por lo cual, los animales con resultados serológicos dudosos o negativos debería repetir la prueba 2-4 semanas después (NovaTec Inmunodiagnostica GmbH 2016). Este aspecto no fue posible en este estudio.

Dado que las muestras analizadas eran de caninos, los anticuerpos, muy probablemente, sean contra Toxocara canis. Sin embargo, puede haber reacción cruzada de anticuerpos contra otros parásitos como: Toxocara cati, Toxascaris leonina, Taenia solium, Ancylostoma caninum y Dipylidium caninum (Smith et al. 1982; Shin \& Liao 2002; Muñoz-Guzmán \& Alba-Hurtado 2010; Silva-Regis et al. 2011; Maizels 2013). Muchos de estos parásitos tienen potencial zoonótico y se pueden transmitir por ruta fecal-oral (Curi et al. 2017); por lo cual, la detección de anticuerpos IgG anti-Toxocara spp., como marcadores de infección, deben alertar sobre la posible presencia de fuentes de contagio para animales y personas. 
Rev. Ciencias Veterinarias, Vol. 38, N 1, [28-42], E-ISSN: 2215-4507, enero-junio, 2020

DOI: https://doi.org/10.15359/rcv.38-1.3

URL: http://www.revistas.una.ac.cr/index.php/veterinaria/index

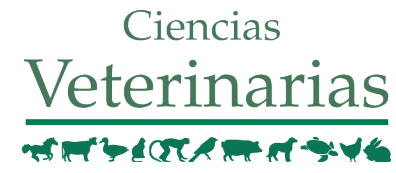

En este estudio, el 75\% de los caninos con anticuerpos anti-Toxocara spp. tenían edades entre 1-6 años. La raza no parece haber influido en el resultado serológico obtenido. Estos resultados concuerdan con lo reportado en perros callejeros de la Ciudad de México. Se considera que los caninos de esas edades tienen mayor probabilidad de realizar caminatas prolongadas y así se exponen más frecuentemente al parásito (Martínez-Barbabosa et al. 2008).

Se conoce poco sobre la interacción de T. canis con el sistema inmune de los caninos. La mayoría de los estudios de este tipo se han documentado en ratones y en humanos (Junginger et al. 2017). Se reporta que los anticuerpos anti-Toxocara spp. persisten entre 3 y 5 años en humanos y, al menos 6 meses en ratones (Fenoy et al. 1992; Chieffi et al. 1995; Fillaux \& Magnaval 2013). No se encontró reportes en la literatura acerca de la duración de los anticuerpos IgG contra este parásito en caninos con infección natural; sin embargo, en perros, experimentalmente infectados, se han mantenido constantes los niveles de esta inmunoglobulina durante al menos 4 meses (Romasanta et al. 2004).

En el grupo de caninos positivos, se identificó tres hembras adultas sin castrar. Una preñada e infectada con T. canis, actúa como amplificadora de la infección porque puede contagiar hasta tres camadas consecutivas sin necesidad de reinfección. Los cachorros nacen infectados y excretan huevos del parásito en las heces, a partir de los 21 días de nacidos. La madre también puede desarrollar una infección patente (Schnieder et al. 2011). En infecciones patentes se puede eliminar, diariamente, hasta 15.000 huevecillos por gramo de heces, contaminando ampliamente los suelos (Papini et al. 2012).

Se ha demostrado que los caninos alimentados con comida casera son más propensos a infectarse con este parásito; sin embargo, el tipo de comida, la cocción y manipulación de esta, influye en el riesgo que pueda representar (Silva-Regis et al. 2011). En este estudio, el tipo de alimentación no parece haber influido en los resultados serológicos.

Con base en la encuesta, se evidenció que la mayoría de los propietarios recogían las heces de los perros en su hogar, pero no lo hacían en áreas públicas. Esta mala práctica favorece la contaminación de los suelos con huevos de parásitos, dando origen a focos de contaminación domiciliares y comunales, las cuales representan un riesgo de infección para las personas y otros animales (Nijsse et al. 2016).

En Costa Rica, se ha reportado una prevalencia de T. canis de 6.9\% y $12 \%$ en arena de algunas playas (Paquet-Durand et al. 2007; Castro et al. 2009), 17.2\% en muestras de suelo peri-domiciliar (Monge \& Barrantes 1986) y 14\% en pasto de parques públicos (Paquet-Durand et al. 2007). En otros países, se reportan prevalencias de hasta 77\% en suelo de áreas de recreación (Gétaz-Schaller et al. 2007).

En este estudio, la mayoría de los caninos seropositivos tenían desparasitaciones trimestrales. Sn embargo, en otros estudios no se ha podido demostrar que la desparasitación trimestral, "a ciegas", realmente sea efectiva en reducir la contaminación del ambiente con parásitos gastrointestinales (Nijsse et al. 2016). Los esquemas tradicionales de desparasitación pueden fallar en eliminar las larvas enquistadas en los tejidos, las cuales podrían estar induciendo una respuesta humoral (Fenoy et al. 1992; Fillaux \& Magnaval 2013; Tropical Council for Companion Animal Parasites 2017). Además, las personas encargadas de los perros desconocían el antiparasitario utilizado; algunas nunca habían llevado a sus mascotas al veterinario, por lo cual, esos protocolos de desparasitación resultan cuestionables. 
El porcentaje promedio de seropositivos fue mayor en viviendas con más de tres perros. Conforme aumenta el número de mascotas, en un mismo espacio, se vuelve más desafiante mantener un adecuado manejo de todos los animales. Se aumenta la probabilidad de transmisión de enfermedades contagiosas entre ellos (Stull et al. 2016). Antes de adquirir mascotas, se debe determinar la cantidad de animales que se pueden albergar bajo condiciones sanitarias adecuadas y no sobrepasar ese límite. Las aglomeraciones son un factor de riesgo para la diseminación de enfermedades en cualquier población (Hurley \& Baldwin 2012; American Veterinary Medical Association 2019). Sin embargo, en Costa Rica, la mayoría de los propietarios admite que, al momento de adquirir un perro, no consideraron las responsabilidades que implicaba (World Animal Protection 2016). Esto puede derivar en un cuidado deficiente de las mascotas.

Si bien, algunos perros con bajos o nulos niveles de cuidado resultaron seronegativos, se debe considerar la posibilidad de que estén en una fase temprana de la infección, o que puedan llegar a infectarse si no se mejoran las prácticas de manejo. Por otra parte, animales con altos niveles de cuidado, resultaron positivos, pero hay que contemplar que las vías de infección y los factores de riesgo de contagio son diversos. Por tanto, se debe analizar toda la historia de vida del animal para determinar las variables que incidieron en la infección con Toxocara spp.

El desconocimiento de los poseedores de perros, acerca de lo que son las enfermedades transmisibles de animales a humanos, favorece un bajo compromiso con las buenas prácticas de manejo de las mascotas (Schantz \& Glickman 1983). Tener perros, como mascotas, ha sido determinado por algunos investigadores como un factor de riesgo para la toxocariasis en humanos (Won et al. 2008). Sin embargo, con una tenencia responsable de los animales y buenas prácticas de higiene, se puede tener una convivencia pacífica y segura con las mascotas (Overgauuw 1997).

Si bien, la investigación tiene limitantes en su impacto estadístico, por contar únicamente con 55 sueros y carecer de muestras de heces, es el primer estudio sobre seroprevalencia de toxocariasis en caninos de Costa Rica. Pone en evidencia la necesidad de determinar el estado real de esta zoonosis en el país mediante investigaciones multidisciplinarias, con mayor número de muestras, incluyendo sueros pareados y heces.

Se necesita mayores esfuerzos para transmitir los resultados de este tipo de investigaciones. Educar a la población en la prevención de enfermedades zoonóticas parasitarias, sobre todo cuando no se tiene acceso a la atención veterinaria regular, aplicando el concepto de "Una salud" para el mejoramiento de la salud pública, animal y ambiental (Organización Mundial de la Salud 2017).

\section{Conclusiones}

La detección de anticuerpos IgG anti-Toxocara spp. es un método valioso como marcador de infección con este parásito en poblaciones caninas. El estudio demuestra que uno de cada tres perros ha tenido contacto, en algún momento, con este nemátodo zoonótico. Además, se encontró hembras seropositivas, sin castrar, que podrían amplificar la infección.

Los dueños de mascotas desconocen qué son las enfermedades zoonóticas. La no recolección de heces en áreas públicas constituyó una de las prácticas de manejo más desatendida. Es indispensable promover el trabajo multidisciplinario y la educación continua de los profesionales en salud y la población, para prevenir la toxocariasis y otras enfermedades parasitarias zoonóticas. 


\section{Agradecimientos}

Los autores desean agradecer al señor Sergio Alfaro, por su ayuda en el procesamiento de los sueros analizados y al Dr. Bernardo Vargas, por su ayuda en el cálculo del margen de error de este estudio, así como su asesoría en el análisis estadístico de los datos.

\section{Conflicto de intereses}

Los autores declaran no tener conflicto de intereses.

\section{Referencias}

American Veterinary Medical Association. 2019. Guidelines for Responsible Pet Ownership. https://www.avma. org/KB/Policies/Pages/Guidelines-for-Responsible-Pet-Ownership.aspx (Accedido 25 de julio 2019).

Arguedas-Zeledón, D., Bitter, E., De Oliveira, J. \& Romero, J.J. 2006. Prevalencia de Toxocara canis y otros parásitos gastrointestinales en perros atendidos en una clínica veterinaria en San José, Costa Rica. Cienc. Vet. 24(2): 137-150.

Bonilla, M., Herrero-Acosta, M.V., Urbina-Villalobos, A. \& Dolz, G. 2018. Detección de anticuerpos contra Trypanosoma cruzi en perros de Costa Rica. Cienc. Vet. 36(2): 1-14. doi: 10.15359/rcv.36-2.1

Calderón, S., De Oliveira, J., Hernández, J., Jiménez, M. \& Muñoz, P. 2008. Parásitos gastrointestinales en caninos menores de seis meses comercializados en tiendas de mascotas de la Gran Área Metropolitana de Costa Rica. Cienc. Vet. 26(1): 21-35.

Castro, C., De Oliveira, J.B., Hernández, J., Jiménez, A. \& Jiménez, M. 2009. Contaminación por parásitos gastrointestinales de caninos en dieciocho playas del Pacífico Central de Costa Rica: implicaciones para la salud pública. Cienc. Vet. 27 (2): 47-56.

Centers for Disease Control and Prevention. 2017. Parasites- Neglected Parasitic Infections (NPIs). https://www.cdc.gov/parasites/npi/index.html (Accedido 14 de diciembre 2018).

Center for Food Security \& Public Health. 2016. Toxocariasis. http://www.cfsph.iastate.edu/Factsheets/ pdfs/toxocariasis.pdf (Acceso: 12 de enero de 2019).

Chieffi, P.P., Peres, B.A., de Mello E.O., Kanamura, H. \& Brandão, M.M. 1995. Persistence of specific antibody response in different experimental infections of mice with Toxocara canis larvae. Rev. Inst. Med. Trop. São Paulo. 37(3): 187-90. doi: 10.1590/s0036-46651995000300001

Curi, N.H.A., Paschoal, A.M.O., Massara, R.L., Santos, H.A., Guimarães, M.P., Passamani, M. \& Chiarello, A.G. 2017. Risk factors for gastrointestinal parasite infections of dogs living around protected areas of the Atlantic Forest: implications for human and wildlife health. Braz. J. Biol. 77(2): 388-395. doi: 10.1590/1519-6984.19515

European Scientific Counsel Companion Animal Parasites. 2017. Worm control in dogs and cats. https://www.esccapuk.org.uk/link-document/20/ (Accedido 25 de julio 2019). 
Fenoy, S., Cuéllar, C., Aguila, C. \& Guillén, J.L. 1992. Persistence of immune response in human toxocariasis as measured by ELISA. Int. J. Parasitol. 22(7): 1037-8. doi: 10.1016/0020-7519(92)90067-U

Fernández, D., De Oliveira, J.B., Calderón, S. \& Romero, J.J. 2008. Prácticas de diagnóstico y control de parásitos de caninos y felinos en 50 clínicas veterinarias del área metropolitana de Costa Rica. Cienc. Vet. 26(2): 51-71.

Fillaux, J. \& Magnaval, J.F. 2013. Laboratory diagnosis of human toxocariasis. Vet. Parasitol. 193(4): 327-336. doi: 10.1016/j.vetpar.2012.12.028

García, L.D., López, M.A., Laffont, H.M., Bojanich, M.V. \& Martín, U. 2014. Seroprevalencia de Toxocara canis en perros de las ciudades de Corrientes y Esperanza (Argentina). Rev. Vet. 25(2): 131-134. doi: $10.30972 /$ vet.252508

Gétaz-Schaller, L., Samalvides-Cuba, F., Breña-Chavez, J., Torrejon, D. \& Maguiña-Vargas, C. 2007. Relación entre toxocariosis y asma: estudio prospectivo en niños del Hospital Nacional Cayetano Heredia, Lima, Perú. Acta Med. Per. 24(2): 81-90.

Hurley, K.F. \& Baldwin, C.J. 2012. Defining capacity/managing population density. In: Greene, C.E. Infectious diseases of the dog and the cat, $4^{\text {th }}$ Edition. Elsevier Saunders, Missouri. p. 1125.

Instituto de Desarrollo Rural de Costa Rica. 2016. Informe de Caracterización Básica Territorio BarvaSanta Bárbara-San Isidro-San Rafael-Santo Domingo-Vara blanca. https://www.inder.go.cr/ heredia-rural/Caracterizacion-Barva-Santa-Barbara-San-Isidro-San-Rafael-Santo-DomingoVara-blanca.pdf (Accedido 14 de diciembre 2018).

Instituto Nacional de Estadística y Censos. 2018. Costa Rica en cifras. http://www.inec.go.cr/sites/default/ files/documetos-biblioteca-virtual/recostaricaencifras2018.pdf (Accedido 14 de diciembre 2018).

Junginger, J., Raue, K., Wolf, K., Janecek, E., Stein, V., Tipold, A., Günzel-Apel, A., Strube, C. \& Hewicker-Trautwein, M. 2017. Zoonotic intestinal helminths interact with the canine immune system by modulating $\mathrm{T}$ cell responses and preventing dendritic cell maturation. Sci. Rep. 7(1): 10310. doi: 10.1038/s41598-017-10677-4

Maizels, R.M. 2013. Toxocara canis: Molecular basis of immune recognition and evasion. Vet. Parasitol. 193(4): 365-374. doi: 10.1016/j.vetpar.2012.12.032

Martínez-Barbabosa, I., Gutiérrez-Quiroz, M., Ruiz-González, L.A., Gutiérrez-Cárdenas, E.M., SosaEdubiel, A.A, Valencia-Juárez, J.L. \& Gaona, E. 2008. Prevalence of anti-T. canis antibodies in stray dogs in Mexico City. Vet. Parasitol. 153(3-4): 270-276. doi: 10.1016/j.vetpar.2008.02.011

Monge, E. \& Barrantes, M. 1986. Prevalencia de huevecillos de Toxocara spp. en los alrededores de la ciudad de San José. Rev. Cost. Cienc. Med. 7(4):339-342.

Municipalidad de Barva. 2014. Plan de desarrollo humano local de Barva. https://munibarva.go.cr/ wp-content/documentos/Planes\%20Anuales\%200perativos/PLAN_DE_DESARROLLO HUMANO_LOCAL.pdf(Accedido 14 de diciembre 2018).

Muñoz-Guzmán, M.A. \& Alba-Hurtado, F. 2010. Antígenos de secreción-excreción de Toxocara canis reconocidos por cachorros del área metropolitana de la Ciudad de México. Vet. Mex. 41(1): 59-64. 
Rev. Ciencias Veterinarias, Vol. 38, Nº 1, [28-42], E-ISSN: 2215-4507, enero-junio, 2020

DOI: https://doi.org/10.15359/rcv.38-1.3

Ciencias

URL: http://www.revistas.una.ac.cr/index.php/veterinaria/index

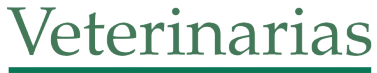

Nijsse, R., Mughini-Gras, L., Wagenaar, J.A. \& Ploeger, H.W. 2016. Recurrent patent infections with Toxocara canis in household dogs older than six months: a prospective study. Parasites \& Vectors. 9(531). doi: 10.1186/s13071-016-1816-7

NovaTec Inmunodiagnostica GmbH. 2016. VetLine Toxocara ELISA. http://www.meditecno.pt/Upload/ Product/Archive/TOCVT0450\%20engl,dt,es-12092016-CS.PDF (Acceso: 02 de agosto de 2018).

Overgauuw, P. 1997. Aspects of Toxocara Epidemiology: Toxocarosis in Dogs and Cats. Crit. Rev. Microbiol. 23(3):233-251. doi: 10.3109/10408419709115138

Organización Mundial de la Salud. 2017. El enfoque multisectorial de la OMS «Una salud». https:// www.who.int/features/qa/one-health/es/ (Accedido 05 de febrero 2020).

Papini, R., Campisi, E., Faggi, E., Pini, G. \& Mancianti, F. 2012. Prevalence of Toxocara canis eggs in dog faeces from public places of Florence, Italy. Helminthologia 49(3): 154 - 158. doi: 10.2478/s11687-012-0031-0

Paquet-Durand, I., Hernández, J., Dolz, G., Romero-Zúñiga, J.J., Schnieder, T. \& Epe, C. 2007. Prevalence of Toxocara spp., Toxascaris leonina and Ancylostomidae in public parks and beaches in different climate zones of Costa Rica. Acta Trop. 104(1): 30-37. doi: 10.1016/j.actatropica.2007.06.011

Rojas, P. 2015. Entre 6 y 7 menores pierden parcialmente su visión cada año, al tener contacto con heces de perros. https://archivo.crhoy.com/entre-6-y-7-menores-pierden-parcialmente-su-vision-cadaano-al-tener-contacto-con-heces-de-perros/nacionales/ (Accedido 14 de diciembre 2018).

Rojas-Salamanca, A.C., León-Bustamante, M.C. \& Bustamante-Saavedra, O.R. 2016. Toxocara canis: una zoonosis frecuente a nivel mundial. Rev. Cien. Agri. 13(1): 19-27. doi: 10.19053/01228420.4803

Romasanta, A., Paz-Silva, A., Sánchez-Andrade, R., Suárez, J.L., Panadero, R., Arias, M., Pedreira, J., Díaz, P., Díez-Baños, P. \& Morrondo, P. 2004. Antibody-mediated response in dogs experimentally infected with Toxocara canis: effect of procodazole. Helminthologia 41(1): 3-7.

Sáenz-Ugalde, C. 2013. Parásitos gastrointestinales con carácter zoonótico y evaluación de algunos parámetros del estado de salud en perros de áreas recreativas de Costa Rica. Tesis de Licenciatura. Universidad Nacional, Heredia, Costa Rica.

Schantz, P.M. \& Glickman, L.T. 1983. Ascáridos de perros y gatos: un problema de salud pública y de medicina veterinaria. Bol. Of. Sanit. Panam. 94(6): 571-586.

Schnieder, T., Laabs, E.M. \& Welz, C. 2011. Larval development of Toxocara canis in dogs. Vet. Parasitol. 175(3-4): 193-206. doi: 10.1016/j.vetpar.2010.10.027

Shin, J.W. \& Liao, W.T. 2002. Humoral immune response to Dipylidium caninum infection of stray dogs in Taiwan. Vet. Parasitol. 104(4): 351-356. doi: 10.1016/s0304-4017(01)00651-3

Silva-Regis, S.C., Ribeiro-Mendoca, L., dos Santos-Silva, N., Cavalcante-Datolli, V.C., Alcântara-Neves, N.M. \& Barrouin-Mello, S.M. 2011. Seroprevalence and risk factors for canine toxocariasis by detection of specific IgG as a marker of infection in dogs from Salvador, Brazil. Acta Trop. 120(12): 46-51. doi: 10.1016/j.actatropica.2011.05.011 


\section{Ciencias}

Smith, H.V., Quinn, R., Bruce, R.G. \& Girdwood, R.W. 1982. Development of the serological response in rabbits infected with Toxocara canis and Toxascaris leonina. T. Roy. Soc. Trop. Med. H. 76(1): 89-94. doi: 10.1016/0035-9203(82)90028-1

Stull, J.W., Sherding, R.G., O’Quin, J., Evason, M.D., Kasten, J.I., Hoet, A.E., Burkhard, M.J. \& Weese, J.S. 2016. Infectious Disease in Dogs in Group Settings: Strategies to Prevent Infectious Diseases in Dogs at Dog Shows, Sporting Events, and Other Canine Group Settings. https://vet.osu. edu/sites/vet.osu.edu/files/documents/preventive-medicine/Infectious $\% 20$ Disease $\% 20 \mathrm{in} \% 20$ Dogs\%20Final.pdf (Accedido 05 de febrero 2020).

Tropical Council for Companion Animal Parasites. 2017. Guidelines for the diagnosis, treatment and control of canine endoparasites in the tropics, $1^{\text {st }}$ Edition. TroCCAP. p. 6-8.

Watthanakulpanich, D. 2010. Diagnostic trends of human toxocariasis. J. Trop. Med. Parasitol. 33 (1): 44-52.

Willard, M.D. 2014. Disorders of intestinal tract. In: Nelson R \& Couto C.G. (Ed.). Small animal internal medicine, $5^{\text {th }}$ Edition. Elsevier Mosby, Missouri. p. 466.

Won, K.Y., Kruszon-Moran, D., Schantz, P.M. \& Jones, J.L. 2008. National seroprevalence and risk factors for zoonotic Toxocara spp. infection. Am. J. Trop. Med. Hyg. 79(4): 552-557.

World Animal Protection. 2016. Estudio nacional sobre tenencia de perros en Costa Rica. https://issuu. com/wspalatam/docs/estudioperros-web-singles (Accedido 14 de diciembre 2018).

Yuill, C. 2011. Wellness examination in dogs. https://vcahospitals.com/know-your-pet/wellnessexamination-in-dogs (Accedido 25 de julio 2018). 